\title{
Razón instantánea de cambio con magnitudes infinitamente pequeñas: una experiencia de Enseñanza a Distancia
}

\author{
Guadalupe Candelario Félix Sandoval \\ Agustín Grijalva Monteverde
}

\begin{abstract}
Resumen: Se muestra un análisis epistemológico utilizando herramientas del Enfoque Ontosemiótico de la Enseñanza y el Conocimiento Matemáticos (EOS), del resultado de una puesta en escena durante la contingencia originada del SARS-CoV-2, la cual fue implementada con alumnos de carreras de ingeniería de una universidad mexicana, mediante una plataforma de videoconferencias para la enseñanza de la razón instantánea de cambio utilizando cantidades infinitamente pequeñas. Se tomaron en cuenta la caracterización de objetos matemáticos, de configuraciones didácticas y, con base en los criterios de idoneidad realizamos un estudio descriptivo del funcionamiento del diseño realizado. El propósito de nuestra propuesta es, diseñar actividades didácticas distintas a las que reposan en la noción de límite que propone la enseñanza tradicional, y presentar actividades más intuitivas, basadas en la noción de magnitud infinitamente pequeña.
\end{abstract}

Palabras clave: Cálculo diferencial. Razón instantánea de cambio. Magnitud infinitamente pequeña.

Guadalupe Candelario Félix Sandoval Maestro en Ciencias con Especialidad en Matemática Educativa por la Universidad de Sonora, México. http://orcid.org/0000-0002-7337-9782 $\bowtie$ guadafeliix@hotmail.com

Agustín Grijalva Monteverde Doctor en Ciencias con Especialidad en Matemática Educativa por el Instituto Politécnico Nacional, México. Profesor asociado de la Universidad de Sonora, México. http://orcid.org/0000-0003-0306-5945 $\triangle$ gutygri1@gmail.com

Recebido em 01/06/2021 Aceito em 22/06/2021 Publicado em 17/07/2021

\section{Instantaneous rate of change with infinitely small magnitudes: a Distance Learning experience}

Abstract: An epistemological analysis is shown using tools from the Ontosemiotic Approach to Mathematical Teaching and Knowledge (EOS), of the result of a staging during the contingency originating from SARS-CoV-2, which was implemented with engineering students from a Mexican university, using a videoconferencing platform for teaching the instantaneous rate of change using infinitely small amounts. The characterization of mathematical objects and didactic configurations were taken into account and, based on the suitability criteria, we carried out a descriptive study of the operation of the design carried out. The purpose of our proposal is to design educational activities other than those that rely on the notion of limit proposed by traditional teaching, and to present more intuitive activities, based on the notion of infinitely small magnitude.

Keywords: Diferential calculus. Instantaneous ratio of change. Infinitely small magnitude.

\section{Taxa de variação instantânea com magnitudes infinitamente pequenas: uma experiência no Ensino à Distância}

\begin{abstract}
Resumo: É apresentada uma análise epistemológica a partir de ferramentas da Abordagem Ontosemiótica ao Ensino e Conhecimento Matemático (EOS), do resultado de uma aula durante a contingência originada do SARS-CoV-2, que foi implementada com estudantes de engenharia de uma universidade mexicana, utilizando uma plataforma de videoconferência para ensinar a taxa de variação instantânea usando quantidades infinitamente pequenas. Levou-se em consideração a caracterização de objetos matemáticos e configurações didáticas e, com base nos critérios de adequação, foi realizado um estudo descritivo do funcionamento do projeto realizado. 0 objetivo da nossa proposta é projetar atividades educacionais diferentes daquelas que se baseiam na noção de limite proposta pelo ensino tradicional, e apresentar atividades mais intuitivas, baseadas na noção de magnitude infinitamente pequena.
\end{abstract}

Palavras-chave: Cálculo diferencial. Taxa de vairação instantânea. Magnitude infinitamente pequena. 


\section{Introducción}

Con base en la experiencia empírica en clases de ingeniería se tuvo una percepción de las diversas dificultades en el Cálculo, entre ellas la de consolidar significados de los objetos matemáticos que intervienen y emergen dentro del curso, aplicar estos significados para la resolución de problemas extramatemáticos, entre otros. En particular, el enfoque de límite en el tratamiento de la derivada es una de las dificultades recurrentes entre los estudiantes. Se revisaron trabajos de investigación, constatándose en diversas fuentes que las percepciones formadas tenían sustento en diversos ámbitos escolares.

En este trabajo se presenta el análisis epistémico de dos secuencias didácticas de un total de seis que se diseñaron para la enseñanza de la razón instantánea de cambio con un enfoque infinitesimal, dirigida a estudiantes del curso "Cálculo Diferencial e Integral l" del área de Ingeniería de la Universidad de Sonora, cuyo propósito es promover la construcción del significado de la razón instantánea de cambio como una variación infinitesimal, a través de la resolución de problemas de contexto tanto extramatemático como intramatemático.

Las secciones que componen el trabajo son las siguientes: Problemática, Justificación, Referentes Teóricos, Estrategia Metodológica, Análisis a priori, Puesta en Escena, Análisis y Conclusiones.

En la sección Problemática y Justificación se describió cuál es el problema que se atendió con nuestra propuesta y con base en qué justificamos dicha propuesta; en la sección Referentes Teóricos se describieron las herramientas del marco teórico utilizadas para el desarrollo de nuestra propuesta; en la sección Estrategia Metodológica se mostró la metodología que se aplicó para nuestra propuesta; en Análisis a priori se muestran las trayectorias epistémicas y sus configuraciones; en Puesta en Escena se muestran las hojas de trabajo de los estudiantes; en Análisis mostramos el análisis de las hojas de trabajo con base en la idoneidad epistémica; y por último en conclusiones mostramos las conclusiones finales del trabajo.

A continuación se presentan las ideas que consideramos importantes de nuestro trabajo: Magnitudes infinitamente pequeñas, Enfoque infinitesimal, Metodología descriptiva, Idoneidad epistémica. 


\section{Problemática}

Con bases empíricas en tanto estudiante en Ingeniería en Mecatrónica, podemos declarar que existen diversas dificultades para construir y comprender los objetos matemáticos involucrados, ya sea como intervinientes o emergentes en el curso de Cálculo Diferencial e Integral I. Un ejemplo de ello es la derivada, que más allá de las distintas formas de tratar a este objeto, en el curso de Cálculo Diferencial e Integral I en carreras de ingeniería se aborda de manera algorítmica utilizando el enfoque de límite, cuya única finalidad es el desarrollo de prácticas centradas en la manipulación algorítmica y mecanizada por medio de las reglas de derivación. Esta manera limita considerablemente al estudiante para formarse un significado de un objeto matemático de manera completa.

En el tratamiento de la derivada, nuestro trabajo se centró en la razón instantánea de cambio, el cual es un tema que se trata con poca profundidad en el currículo y no se le otorga la relevancia correspondiente en el plan de estudios.

Por lo tanto, la problemática principal en la cual se ubica este trabajo corresponde al diseño de propuestas alternativas para la enseñanza de la razón instantánea de cambio, con un enfoque distinto al acercamiento mediante el uso de límites, cuyas complicaciones para el aprendizaje han sido documentadas ampliamente, tema del que aportamos algunos elementos en el apartado siguiente.

\subsection{Dificultades de los estudiantes en el estudio del cálculo}

Artigue (1995) describe una serie de problemas relacionados al concepto límite de diferentes naturalezas, de los cuales se destacan los siguientes:

- Los problemas epistemológicos, que son aquellos conocimientos que, en lugar de ayudar a construir de una manera sólida el concepto, solo entorpecen su construcción.

- El sentido que evoca el término límite, es decir que hace pensar al alumno como una barrera intraspasable y no alcanzable. Con estas características se puede concluir que de ninguna forma el concepto límite puede catalogarse como un concepto intuitivo.

- En el sentido geométrico, el concepto de límite pierde claridad por la forma en que el proceso se lleva a cabo.

- En el carácter histórico sucede algo parecido a los problemas epistemológicos, ya que entorpecen al desarrollo de la construcción del concepto. 
- En la formulación de preguntas que podrían evocar al concepto límite tiene posibilidades de tergiversar conceptos, incluso contrarios, como las sucesiones dinámicas y un número finito.

- Mencionar que el concepto límite es una proximidad o un área vecina quebranta solidez en el concepto y a su vez genera dificultades por el mal manejo del criterio.

También existen complicaciones para concebir el concepto límite mediante la transición de prácticas del álgebra a prácticas del cálculo, ya que la facilidad de identificar relaciones del tipo numérico/algebraico no son comparables a las relaciones álgebra/cálculo.

\section{Justificación}

El trabajo de Cornu (1981) nos proporciona una aproximación hacia la percepción que tienen los alumnos sobre el concepto de límite, con base en las ideas construidas por su enseñanza tradicional. Este autor señala que en el lenguaje común los estudiantes utilizan la idea de límite desde un punto de vista geométrico, donde el límite es algo que no se sobrepasa o se alcanza. Sostiene también que la expresión "tender hacia" no forma parte del lenguaje natural construido por el alumno, ya que no termina de construir el concepto, es decir, para al alumno es difícil utilizar frases como: "Ese amarillo tiende hacia el verde" o "Los trabajos de esa persona tienden a un premio Nobel", porque para el alumno el límite se define como una cantidad numérica que jamás se logra alcanzar. Una consecuencia de esto es que, para algunos, en la noción del límite no está intrínseca la idea de variación, movimiento, ni de aproximación a dicho límite.

Por lo anterior, el autor concluyó que los alumnos de enseñanza media no alcanzan a construir definiciones formales en términos del límite, por lo que no les es posible crear articulaciones entre una definición y alguna otra representación de dicho concepto.

Por otra parte, el trabajo de Quesada Barrioseta (2014) trata a la derivada como objeto de estudio de situaciones del día a día, como la velocidad de una motocicleta, entre otros fenómenos, con un método constructivista.

Gonzales, y Radillo (2014) elaboraron una propuesta para la enseñanza del concepto de derivada de una función mediante actividades de visualización.

Schivo, Sgreccia, y Caligaris (2014) hablan de la visualización de la derivada, pero implementando el uso de la tecnología, en el que se pretende dar un acercamiento diferente al método tradicional. 
Aunque los acercamientos son diferentes, se basan en la idea de límite, el cual genera numerosas complicaciones y como propuesta diferente, que consideramos más intuitiva en este trabajo se propone tratar con las magnitudes infinitamente pequeñas.

\subsection{Enfoque infinitesimal}

Para la introducción de las cantidades infinitamente pequeñas, primero se diseñaron actividades para introducir magnitudes o cantidades relativamente "muy pequeñas" con relación a otras 0, equivalentemente, "muy grandes" con relación a otras. Por ejemplo, una micra es "muy pequeña" con relación a la distancia entre la tierra y júpiter, de tal suerte que si sumamos, por ejemplos, la distancia señalada más una micra, para efectos prácticos, una micra no aporta nada a la suma. Si tomamos ahora el cuadrado de una micra, obtenemos cantidades aún "más pequeñas" y así sucesivamente.

Con base en esas ideas, introducimos la noción de magnitud infinitamente pequeña, retomando infinitesimales de J.L. Bell (1998) quien señala que una magnitud $\delta$ es infinitesimal si cumple las siguientes características:

$-\delta \neq 0$.

- si $\delta>0$, entonces $\delta$ es más pequeño que cualquier número real positivo.

- si $\delta<0$, entonces $\delta$ es mayor que cualquier número real negativo.

- $\delta^{2}=0$ (y, por lo tanto, todas las potencias superiores de $\delta$, como $\delta^{3}$ y $\delta^{4}$, también son $0)$.

Por las propiedades atribuidas a las magnitudes infinitesimales puede observarse que no son números reales y hacer un estudio formal de los mismos escapa a las intenciones y alcances del presente trabajo.

La definición anterior muestra que los infinitesimales son magnitudes que están más cerca de 0 que cualquier otro número positivo o negativo sin ser ellos mismos cero, y elevándolos a potencias mayores que o igual a 2 los hace 0 . Entonces los infinitesimales no son números reales. Debe resaltarse que en el tratamiento tradicional del cálculo también se hace referencia a nociones

\footnotetext{
1 Nota: cualquier infinitesimal multiplicado por un número real distinto de cero también es un infinitesimal, mientras que 0 veces un infinitesimal es 0 .
} 
complicadas como la de infinito, que tampoco es un número real. Uno puede pensar en infinitesimal como un número infinitamente pequeño arbitrariamente cercano a 0 pero sin ser 0 .

La letra $\delta$ tiene un significado polisémico, ya que puede tratarse de una magnitud infinitamente pequeña, pero a su vez como una variación infinitamente pequeña de alguna variable. Cuando se asocia esta letra a alguna variable, entonces esta es tratada como una variación. Por ejemplo, $\delta x$ y $\delta y$.

Como ya se mencionó, $\delta$ no es cero, y otra diferencia a destacar sobre ello es la siguiente: al operar un número natural como el 2 con 0 y un $\delta x$ infinitesimal se obtiene lo siguiente: $2 \cdot 0$ y 0 son iguales, pero $2 \cdot \delta x$ y $\delta x$ son distintos. Esto se mantiene para cualquier constante distinta de cero, no solo con el número 2.

El cociente $\frac{\delta y}{\delta x}$, referido a las variaciones infinitesimales tanto de la variable independiente como de la variable dependiente de una función $y=f(x)$ ahora se puede definir de la siguiente manera:

Sea $\delta x$ un infinitesimal, tal que $f(x+\delta x)$ esté definido. Entonces $\delta y=f(x+\delta x)-f$ $(x)$ también es un infinitesimal, y la derivada de $y=f(x)$ en $x$ es la relación de $\delta y$ a $\delta x: \frac{\delta y}{\delta x}=$ $\frac{f(x+\delta x)-f(x)}{\delta x}$

\section{Referentes teóricos a grandes rasgos}

Los elementos teóricos del Enfoque Ontosemiótico del Conocimiento y la Instrucción Matemáticos (EOS) que utilizamos para el diseño de las actividades didácticas y para la evaluación de nuestra propuesta didáctica en general fueron los siguientes: El significado institucional de referencia y el significado institucional pretendido.

Una aclaración pertinente es la consideración de que el significado institucional de referencia al que hacemos alusión en nuestro trabajo se refiere a la identificación de las problemáticas que se quieren abordar y resolver en el plan de estudios del curso de Cálculo Diferencial e Integral I de las carreras de ingeniería de la Universidad de Sonora, de tal manera que aunque nuestra propuesta se diseña con un enfoque diferente, se promueve la resolución de las mismas problemáticas, particularmente la caracterización y utilización de la noción "razón instantánea de cambio". De cualquier forma señalaremos en la sección correspondiente los objetos matemáticos primarios que se utilizan principalmente para nuestro diseño y cuyo análisis más 
profundo constituye el significado institucional de referencia para el estudio del cálculo mediante el uso de magnitudes infinitamente pequeñas.

Con respecto a los contenidos matemáticos y didácticos utilizamos los indicadores de la idoneidad didáctica y sus dimensiones. De igual manera, la valoración para la propuesta y sus posibles mejoras se hicieron con base en la idoneidad didáctica.

\subsection{Significados}

A continuación se describen por una parte el significado institucional de referencia, en el que se señalan los objetos matemáticos de nuestro interés y la forma en que se abordan los mismos en acercamiento basados en el manejo de las cantidades infinitesimales. Asimismo, se presenta el significado institucional pretendido por el currículo, del cual se toman también, como parte del significado institucional de referencia, las situaciones problema que se proponen que un estudiante pueda resolver al final del curso.

\subsection{Configuración didáctica}

EI EOS analiza esta relación con base en un sistema entre elementos que lo conforman dentro de un trasfondo ecológico. Uno de estos elementos es la configuración epistémica que se asocia a una tarea o práctica, y los elementos primarios como lenguajes, conceptos, proposiciones, procedimientos y argumentos, producidos ya sea parte del profesor, del alumno 0 ambos. Relacionado con la configuración anterior está la configuración instruccional que consiste en el conjunto de objetos por parte del profesor y el alumno así como los objetos mediacionales, que son los recursos materiales con los que cuentan tanto profesor como alumno. Y por último la descripción de la construcción de los aprendizajes que se desarrolla a los largo de las sesiones es consecuencia de una configuración cognitiva, que son el conjunto de objetos previos y emergentes de las prácticas personales puestas en juego durante la implementación de la configuración epistémica antes descrita.

\footnotetext{
"Sistema articulado de roles docentes y discentes, a propósito de una configuración de objetos y procesos matemáticos ligados a una situación - problema, constituye la principal herramienta para el análisis de la instrucción matemática. Las configuraciones didácticas y su secuencia en trayectorias didácticas tienen en cuenta las facetas epistémica (conocimientos institucionales), cognitiva (conocimientos personales), afectiva, mediacional (recursos tecnológicos y temporales), interaccional y ecológica que caracterizan los procesos de estudio matemático." (GODINO et al., 2017, p. 94).
} 
En nuestro trabajo nos enfocaremos en la trayectoria epistémica, la cual será descrita a continuación.

\title{
4.3 Trayectoria epistémica
}

EI EOS cuenta con un modelo cognitivo elaborado de tal manera que distingue seis categorías de entidades primarias que se constituyen de los sistemas de prácticas ya mencionadas previamente, estas entidades primarias son las siguientes: lenguajes, situaciones, acciones, conceptos, proposiciones y argumentos. La trayectoria epistémica es la distribución de los componentes mencionados a través de un proceso de estudio.

\begin{abstract}
"Se distinguen seis categorías de entidades primarias como constituyentes de los sistemas de prácticas: lenguaje, situaciones, acciones, conceptos, proposiciones y argumentos. La trayectoria epistémica es la distribución en el tiempo de estos componentes en un proceso de estudio. Distinguiremos, por tanto, en ellas seis estados posibles, según el tipo de componente del significado sistémico que se estudia en cada momento." (GODINO, 2002, p. 6)
\end{abstract}

- Situacional: se aborda el planteamiento de un ejemplar del tipo de problemas incluidos en el significado.

- Actuativo: se aborda el desarrollo o estudio de una manera de resolver los problemas.

- Notacional: se introducen notaciones, representaciones gráficas, etc.

- Conceptual: se formulan, interpretan o aplican definiciones de los objetos puestos en juego.

- Proposicional: se enuncian, interpretan y aplican propiedades.

- Argumentativo: se justifican las acciones adoptadas o las propiedades enunciadas.

En el estudio de un cierto tema, estos estados del proceso instruccional van emergiendo conforme avanza el estudio.

"El análisis de la trayectoria epistémica (análisis epistémico) de un proceso instruccional permitirá caracterizar el significado sistémico efectivamente implementado. Para analizarla, su desarrollo o crónica será dividido en unidades de análisis, considerando una nueva unidad cuando la trayectoria epistémica cambia de estado, esto es, el estudio se centra en un tipo de entidad primaria diferente." (GODINO, 2002, p. 6). 


\subsection{Idoneidad didáctica}

La idoneidad didáctica es un sistema de indicadores empíricos que permiten reunir ciertas características para calificar como idóneas la implementación de la intervención didáctica, y las prácticas del estudiante. De esta manera se busca una adaptación entre los significados personales logrados por el estudiante y los significados institucionales pretendidos, considerando a su vez las circunstancias y recursos disponibles.

La idoneidad epistémica es aquella que se refiere al grado de representatividad de los significados institucionales implementados (o pretendidos), respecto de un significado de referencia.

Este elemento analiza la diversidad de significados interconectados apoyándose de la diversidad de objetos: Situaciones, lenguajes, conceptos, proposiciones, procedimientos, y argumentos.

Tabla 1: Tabla de indicadores de idoneidad epistémica

\begin{tabular}{|c|l|}
\hline Componentes & \multicolumn{1}{c|}{ Indicadores } \\
\hline $\begin{array}{c}\text { Situaciones } \\
\text { problemas }\end{array}$ & $\begin{array}{l}\text { - Se presenta una muestra representativa y articulada de situaciones de } \\
\text { contextualización, ejercitación y aplicación } \\
\text { - Se proponen situaciones de generación de problemas (problematización) }\end{array}$ \\
\hline Lenguajes & $\begin{array}{l}\text { - Uso de diferentes modos de expresión matemática (verbal, gráfica, } \\
\text { simbólica...), traducciones y conversiones entre los mismas. } \\
\text { - Nivel del lenguaje adecuado a estudiantes a los que se dirige } \\
\text { - Se proponen situaciones de expresión matemática e interpretación }\end{array}$ \\
\hline $\begin{array}{c}\text { Reglas } \\
\text { (Definiciones, } \\
\text { proposiciones, } \\
\text { procedimientos) }\end{array}$ & $\begin{array}{l}\text { - Las definiciones y procedimientos son claros y correctos, y están adaptados } \\
\text { - Se presentan los enunciados y procedimientos fundamentales del tema para } \\
\text { el nivel educativo dado } \\
\text {-Se proponen situaciones donde los alumnos tengan que generar o negociar } \\
\text { definiciones proposiciones o procedimientos }\end{array}$ \\
\hline Argumentos & $\begin{array}{l}\text { - Las explicaciones, comprobaciones y demostraciones son adecuadas al } \\
\text { nivel educativo a que se dirigen } \\
\text {-Se promueven situaciones donde el alumno tenga que argumentar }\end{array}$ \\
\hline Relaciones & $\begin{array}{l}\text { Los objetos matemáticos (problemas, definiciones, proposiciones, etc.) se } \\
\text { relacionan y conectan entre sí. } \\
\text { - Se identifican y articulan los diversos significados de los objetos que } \\
\text { intervienen en las prácticas matemáticas. }\end{array}$ \\
\hline
\end{tabular}

Fuente: Godino (2013)

\section{Estrategia metodológica}

Un trabajo como el presente, centrado en el diseño de actividades didácticas para la enseñanza y el aprendizaje no sigue propiamente las metodologías empleadas en los procesos de investigación, pero tampoco le son ajenos, pues al someter el diseño a experimentación, en los 
hechos se realiza algún tipo de investigación y eso nos motiva a decidir elementos mínimos a considerar para esta fase experimental.

Lo primero que debemos destacar es que el propósito de la investigación no consiste en profundizar en el conocimiento de un determinado fenómeno u objeto, sino, partiendo de lo que se asume como construido por la comunidad que nos sirve de referencia se procede a hacer el diseño (con elementos epistémicos, cognitivos, y otros) y la experimentación busca detectar las deficiencias e inconsistencias del diseño, para tener elementos que contribuyan a mejorar el mismo, con base en los análisis que el marco teórico seleccionado nos permite hacer.

Sin embargo, nos parece que la metodología de investigación proporciona directrices que pueden retomarse para hacer los análisis de la fase experimental con el propósito de mejorar el diseño de las actividades didácticas, pues el diseño se hizo a partir de análisis preliminares que vaticinaban el posible éxito de su aplicación y a partir de lo sucedido en la fase experimental se hizo un análisis a posteriori para contrastarlo con el análisis preliminar, con el propósito, insistimos, no de profundizar en el conocimiento del fenómeno de estudio, sino de mejorar el diseño.

Con estas consideraciones, se decidió que lo más conveniente para tener elementos de análisis era hacer un estudio basado en los criterios de la idoneidad didáctica propuestos en el EOS, con lo cual estamos en condiciones de hacer un estudio descriptivo del funcionamiento del diseño de actividades didácticas.

En Hernández (2006, p. 102) se dice que:

\begin{abstract}
Con frecuencia, la meta del investigador consiste en describir fenómenos, situaciones, contextos y eventos; esto es, detallar cómo son y se manifiestan. Los estudios descriptivos buscan especificar las propiedades, las características y los perfiles de personas, grupos, comunidades, procesos, objetos o cualquier otro fenómeno que se someta a un análisis (Danhke, 1989). Es decir, miden, evalúan o recolectan datos sobre diversos conceptos (variables), aspectos, dimensiones o componentes del fenómeno a investigar. En un estudio descriptivo se selecciona una serie de cuestiones y se mide o recolecta información sobre cada una de ellas, para así (valga la redundancia) describir lo que se investiga.
\end{abstract}

Precisamente el análisis de la idoneidad didáctica, a partir de los indicadores usados, conducen a hacer un análisis exhaustivo de carácter descriptivo de la práctica educativa en el aula, de un texto, de una secuencia didáctica u otros aspectos educativos.

Las razones expuestas nos conducen a aplicar los criterios de idoneidad didáctica señalados con la finalidad de hacer un estudio descriptivo tanto del diseño de las secuencias didácticas, como de los resultados contenidos al llevarlos a la práctica. También revisamos ciertos 
materiales que por su temática ofrecían acercamientos de utilidad para el diseño de nuestras actividades, como por ejemplo los trabajos Verón (2021) y Godino (2021).

\section{Análisis a priori (Trayectorias epistémicas de las secuencias)}

Es de utilidad analizar las trayectorias epistémicas de cada secuencia, con el fin de ubicar los objetos matemáticos primarios que se espera deberán emerger al resolver las diferentes situaciones problemas planteados.

Para hacer dichas trayectorias epistémicas se tomaron las diferentes actividades y se organizaron en las diferentes configuraciones epistémicas, con lo cual se resalta el carácter fundamental que se pretende desarrollar en un conjunto de actividades.

Tabla 2: Trayectoria epistémica de la secuencia didáctica 1

\begin{tabular}{|c|c|c|}
\hline $\begin{array}{c}\text { Configuraciones } \\
\text { epistémicas }\end{array}$ & Descripción & Estado \\
\hline CE1 & Planteamiento del problema extramatemático & Situacional \\
\hline CE2 & $\begin{array}{l}\text { Descripción subjetiva del papel propio sobre el } \\
\text { problema }\end{array}$ & Argumentativo \\
\hline CE3 & $\begin{array}{l}\text { Uso de la notación " } k g \text { " para la identificación de } \\
\text { kilogramos y "ton" para la tonelada }\end{array}$ & Proposicional \\
\hline CE4 & $\begin{array}{l}\text { Aplicación de cocientes para la conversión de } \\
\text { toneladas a kilogramos }\end{array}$ & Actuativo \\
\hline CE5 & $\begin{array}{l}\text { Aplicación de cocientes para el cálculo de } \\
\text { kilogramos de basura por habitante en el mundo }\end{array}$ & Actuativo \\
\hline CE6 & $\begin{array}{l}\text { Aplicación de la "regla de tres" para el cálculo de } \\
\text { porcentaje de la repercusión de una persona en } \\
\text { la contaminación mundial }\end{array}$ & Actuativo \\
\hline CE7 & $\begin{array}{l}\text { Distinción de cantidades, entre una cantidad } \\
\text { pequeña y el cero. }\end{array}$ & Argumentativo \\
\hline
\end{tabular}




\begin{tabular}{|c|c|c|}
\hline CE8 & $\begin{array}{l}\text { Distinción entre una cantidad pequeña y otra de } \\
\text { magnitud casi igual. }\end{array}$ & Argumentativo \\
\hline CE9 & $\begin{array}{llll}\begin{array}{l}\text { Planteamiento del } \\
\text { extramatemático }\end{array} & & & \\
& & \\
\end{array}$ & Situacional \\
\hline CE10 & Aplicación de cocientes entre cantidades grandes & Actuativo \\
\hline CE11 & $\begin{array}{l}\text { Aplicación de productos de cantidades grandes } \\
\text { con grandes y de cantidades grandes con } \\
\text { pequeños }\end{array}$ & Actuativo \\
\hline CE12 & $\begin{array}{l}\text { Aplicación de cocientes cuando una cantidad es } \\
\text { grande. }\end{array}$ & Actuativo \\
\hline CE13 & Suma cuando una cantidad es pequeña. & Actuativo \\
\hline CE14 & $\begin{array}{l}\text { Utilización del símbolo } \approx \text { para denotar que es la } \\
\text { aproximación de una cantidad. }\end{array}$ & Proposicional \\
\hline CE15 & $\begin{array}{l}\text { Utilización de calculadora para obtener la suma } \\
\text { de cantidad natural y pequeña. }\end{array}$ & Actuativo \\
\hline CE16 & Aplicación de cociente entre cantidades grandes. & Actuativo \\
\hline CE17 & $\begin{array}{l}\text { Aplicación de cociente cuando una cantidad es } \\
\text { grande. }\end{array}$ & Actuativo \\
\hline CE18 & $\begin{array}{l}\text { Aplicación de producto cuando una cantidad es } \\
\text { pequeña. }\end{array}$ & Actuativo \\
\hline CE19 & $\begin{array}{l}\text { Graficación de porcentajes anteriormente } \\
\text { calculados. }\end{array}$ & Notacional \\
\hline CE2O & $\begin{array}{l}\text { Descripción de rasgos esenciales para la } \\
\text { identificación de cantidades grandes y pequeñas }\end{array}$ & Conceptual \\
\hline CE21 & $\begin{array}{l}\text { Planteamiento de situaciones para su } \\
\text { comparación }\end{array}$ & Situacional \\
\hline CE22 & $\begin{array}{l}\text { Diferenciación de tamaño entre cantidades por } \\
\text { medio de situaciones diversas }\end{array}$ & Actuativo \\
\hline
\end{tabular}




\begin{tabular}{|c|l|l|}
\hline CE23 & $\begin{array}{l}\text { Planteamiento de situación con base en la } \\
\text { diferencia de cantidades }\end{array}$ & Situacional \\
\hline CE24 & $\begin{array}{l}\text { Análisis de situaciones con base en el tamaño de } \\
\text { diferencias planteadas }\end{array}$ & Actuativo \\
\hline CE25 & $\begin{array}{l}\text { Planteamiento de una situación con base en la } \\
\text { masa de un átomo }\end{array}$ & Situacional \\
\hline CE26 & Operación con cantidades pequeñas & Actuativo \\
\hline CE27 & $\begin{array}{l}\text { Una cantidad dada siempre se puede hacer más } \\
\text { pequeña si ésta es real }\end{array}$ & Proposicional \\
\hline CE28 & $\begin{array}{l}\text { Distinción entre cantidades muy pequeñas e } \\
\text { infinitamente pequeñas }\end{array}$ & Argumentativo \\
\hline CE29 & $\begin{array}{l}\text { Las cantidades infinitamente pequeñas no se } \\
\text { pueden hacer más pequeñas }\end{array}$ & Proposicional \\
\hline
\end{tabular}

Fuente: Félix, G. (2020)

En la actividad de inicio se plantea una situación problema referente a la contaminación mundial (CE1). La primer serie de preguntas tiene como propósito que el estudiante se problematice con el contexto y argumente la repercusión de su papel en dicho contexto (CE2). La segunda serie de preguntas establece una relación de magnitudes proporcionales con referencia a la situación problema para realizar conversiones entre magnitudes utilizando la regla de tres (CE3, CE4, CE5, CE6). La tercera serie de preguntas tiene como propósito que el estudiante argumente las diferencias entre los cocientes calculados de la serie de preguntas previa (CE7, CE8). En la actividad de desarrollo, se plantea una segunda situación problema con referencia a la contaminación y reciclaje en México (CE9). En la primera serie de preguntas, el estudiante calcula cocientes de cantidades grandes y pequeñas para obtener diferentes porcentajes (CE10, CE11, CE12), realiza sumas entre porcentajes para observar la repercusión que tienen las cantidades pequeñas en cantidades más grandes, sin tecnología y con tecnología, en este caso una calculadora científica (CE13, CE14, CE15), y calcula cocientes entre cantidad grande y pequeña, cantidad pequeña y grande, y cantidades naturales (CE16, CE17, CE18). Después, se presenta una recta numérica de porcentajes para que el estudiante represente en ella todos los porcentajes calculados previamente (CE19). En la actividad de cierre, se establece la relatividad de las cantidades según sea su comparación (CE20). En el primer inciso de esta actividad el 
estudiante identifica el tamaño de diferencias entre dos números (CE21, CE22). En el segundo inciso la dinámica se torna al revés, por medio de indicadores, deben plantear situaciones (CE23, CE24). Por último, en el tercer inciso se parte desde el planteamiento del peso de un protón, para enseguida pasar a cuestionar si un número tan pequeño, puede ser más pequeño (CE25, CE26). Se declara también que siempre que se trata de un número real, éste puede ser tan pequeño como se desee (CE27). Y para culminar la actividad se plantean magnitudes infinitamente pequeños y se discute la posibilidad de poder hacerlos más pequeños de alguna manera (CE28, CE29).

Tabla 3: Trayectoria epistémica de la secuencia didáctica 2

\begin{tabular}{|c|c|c|}
\hline $\begin{array}{c}\text { Configuraciones } \\
\text { epistémicas }\end{array}$ & Descripción & Estado \\
\hline CE1 & $\begin{array}{l}\text { Representación de gráfica de números reales } \\
\text { mayores a cero. }\end{array}$ & Notacional \\
\hline CE2 & $\begin{array}{l}\text { Identificación y graficación de números en la recta } \\
\text { numérica. }\end{array}$ & $\begin{array}{ll}\text { Actuativo } \quad y \\
\text { Notacional }\end{array}$ \\
\hline CE3 & $\begin{array}{l}\text { Planteamiento de que "no existe un número real } \\
\text { que sea el más pequeño". }\end{array}$ & Proposicional \\
\hline CE4 & $\begin{array}{l}\text { Formalización de las magnitudes infinitesimales } \\
\text { como magnitud más pequeñas que cualquier } \\
\text { número real positivo. }\end{array}$ & Conceptual \\
\hline CE5 & $\begin{array}{l}\text { Representación de una cantidad infinitesimal en la } \\
\text { recta numérica. }\end{array}$ & Notacional \\
\hline CE6 & $\begin{array}{l}\text { Representación gráfica de la adición de un número } \\
\text { infinitesimal a un número real. }\end{array}$ & Notacional \\
\hline CE7 & La diferencia entre $\delta$ y $n \delta$ no es medible. & Argumentativo \\
\hline CE8 & $\begin{array}{l}\text { Cualquier número real multiplicado por un número } \\
\text { infinitesimal da como resultado un número } \\
\text { infinitesimal } \forall n: n \delta \approx \delta \text {. }\end{array}$ & Proposicional \\
\hline
\end{tabular}




\begin{tabular}{|c|c|c|}
\hline CE9 & $\begin{array}{l}\text { Construcción de la representación gráfica de las } \\
\text { magnitudes infinitesimales. }\end{array}$ & Notacional \\
\hline CE10 & $\begin{array}{l}\text { Introducción de un cuadrado con medidas de lado } \\
l \text {. }\end{array}$ & $\begin{array}{l}\text { Situacional } \\
\text { Notacional }\end{array}$ \\
\hline CE11 & $\begin{array}{l}\text { Relación entre las magnitudes infinitesimales de } \\
\text { las variables dependiente e independiente. }\end{array}$ & Conceptual \\
\hline CE12 & $\begin{array}{l}\text { Representación algebraica y aritmética de } \\
\text { incrementos infinitesimales de las variables. }\end{array}$ & Actuativo \\
\hline CE13 & $\begin{array}{l}\text { Representación geométrica de los incrementos } \\
\text { infinitesimales de las variables. }\end{array}$ & $\begin{array}{l}\text { Notacional } \\
\text { Actuativo }\end{array}$ \\
\hline CE14 & $\begin{array}{l}\text { Los incrementos mínimos siempre serán de } \\
\text { tamaño infinitesimal. }\end{array}$ & Proposicional \\
\hline CE15 & $\begin{array}{l}\text { Diferenciación entre los distintos tipos de } \\
\text { representación de un número infinitesimal según } \\
\text { sea el caso. }\end{array}$ & Proposicional \\
\hline
\end{tabular}

Fuente: Félix (2020)

En la actividad de inicio se plantea una escala unitaria en la recta numérica y se pide graficar en ella ciertos números (CE1, CE2). Se declara con base en la propiedad Arquimediana que todo número real por más pequeño que sea, siempre puede ser más pequeño si se divide entre dos (CE3). En la actividad de desarrollo se introduce la notación formal $\delta$ como representación de los infinitesimales, que son magnitudes más pequeñas que cualquier número real positivo y que al multiplicarse por sí mismos dan como resultado cero (CE4). En el primer inciso se vuelve a graficar números, pero esta vez con $\delta$ y números naturales sumados y multiplicados por $\delta$, así como se induce un argumento acerca de la diferencia medible entre $\delta$ y $n \delta$ (CE5, CE6, CE7). Al término de este inciso se establece la propiedad de que cualquier número real multiplicado por un número infinitesimal da como resultado un número infinitesimal (CE8). En el segundo inciso se grafican números que son productos de naturales con infinitesimales (CE9). En la actividad de cierre se introduce un cuadrado de lado $l$ para contestar diversas preguntas (CE10). Las preguntas planteadas son acerca de incrementos de varios tamaños que sufre la figura antes mencionada, esto con el propósito de hacer notar que un incremento mínimo es infinitamente pequeño, y se supone que esto generará la asociación de $\delta$ con una variable, en un 
contexto algebraico y geométrico (CE11, CE12, CE13, CE14). Al finalizar la secuencia se menciona acerca de los diferentes significados con los que se utiliza el símbolo $\delta$ (CE15).

\section{Puesta en escena}

En esta sección hablaremos acerca de los detalles principales de la puesta en escena de las secuencias didácticas diseñadas por nuestra propuesta, la cual se llevó a cabo con dos grupos de 3 estudiantes cada uno. Trabajamos durante tres días en sesiones de dos a tres horas diarias, por medio de la plataforma Microsoft Teams. Sin embargo, debido al tipo de trabajo, solo describiremos lo sucedido en la primera sesión.

Las actividades de nuestras secuencias se diseñaron de tal manera que los estudiantes pudiesen construir nuevos conocimientos de manera progresiva y estructurada. Es decir, primero diseñamos actividades con las que se pudiesen familiarizar con el entorno, utilizando problemas tanto extramatemáticos como intramatemáticos, para así, poco a poco ir diseñando una trayectoria didáctica hacia la construcción de los infinitesimales y nociones como la razón instantánea de cambio. Como evidencia del trabajo desarrollado, contamos con las hojas de trabajo con las respuestas de los estudiantes participantes.

La puesta en escena se desarrolló de la siguiente manera:

Debido a situaciones que no estaban en nuestras manos, pues la pandemia ocasionada por el coronavirus SARS-COV-2 obligó al confinamiento, era inadecuado hacer trabajo de manera presencial. A consecuencia de ello, la implementación se desarrolló en la plataforma Microsoft Teams. Por ese medio se presentaron las hojas de trabajo de cada estudiante y se realizaron los comentarios e intervenciones pertinentes.

Los grupos que se prestaron para colaborar con nosotros eran pequeños: Dos grupos conformados por 3 estudiantes cada uno. Las sesiones de implementación tenían una duración de dos a tres horas aproximadamente para la resolución de dos secuencias didácticas por día. Cada estudiante contaba con su equipo de cómputo desde su respectiva casa, conexión a internet y el recurso del correo electrónico por el cual hacían llegar sus hojas de trabajo. El diseñador de las actividades fue el encargado de dirigir los equipos de trabajo, realizar las intervenciones necesarias e institucionalizar conceptos para las secuencias didácticas presentes y posteriores. Se realizaron grabaciones simultáneas, una en donde participábamos todos los integrantes de la sesión para atender dudas o dar alguna indicación, y otra donde grabábamos a cada equipo trabajando por separado para un mejor análisis individual de cada equipo. Al terminar una 
secuencia se hacía una pausa para atender los temas abordados dentro de dicha secuencia. El diseñador de las actividades preguntaba dudas, e institucionalizaba de manera breve lo realizado al final de cada sesión.

Durante las sesiones se realizó un proceso guiado por el diseñador de las actividades, de tal manera que cubriera todos los elementos que se consideraban necesarios. Los elementos de las sesiones son los que se muestran a continuación:

1. Un diálogo grupal con el cual se acerca al estudiante al contexto que queremos promover para el desarrollo de las cosas grandes y pequeñas.

2. Provocar una exploración y descubrimiento sobre la existencia de las cantidades infinitamente pequeñas o infinitesimales y las propiedades que a éstas la definen como tal.

3. Utilizar contextos matemáticos para establecer una relación algebraica - geométrica sobre las magnitudes infinitesimales y el símbolo $\delta$.

\subsection{Aspectos destacados}

Durante la puesta en escena pudimos rescatar algunos aspectos importantes, como la aceptación de los estudiantes de los contextos elegidos para los problemas, ya que al elegir la contaminación mundial y la de nuestro país mostraron un interés en las situaciones y alcanzaron a concientizar el impacto ecológico que los humanos causamos en el planeta.

Por otro lado, el no tener conocimientos previos acerca de los infinitesimales provocó que la mayoría tuviese cierta incertidumbre y dudas de muchas cosas, por ejemplo de mantener las propiedades de los números enteros a la hora de operar con magnitudes infinitesimales, o confundirse cuando había que conceptualizarlo gráficamente por no tener muy claro qué era el infinito. Un problema sobresaliente era la frase "algo infinitamente pequeño", ya que ellos decían que era algo que no se podía contar y al no poder contarlo, se les dificultaba trazarlo e incluso tomarlo en cuenta en las operaciones algebraicas.

\subsection{Narración}

Para comenzar con las sesiones, el profesor titular del grupo hizo una especie de presentación para diseñador/conductor de las secuencias, se mencionaron datos generales de los participantes y por medio de la plataforma Teams se hicieron llegar las hojas de trabajo. Una vez con las hojas listas, se dieron las indicaciones de leer las hojas detenidamente y contestarlas de 
manera amplia. Se tomó el tiempo de inicio para llevar un control de la duración, y esperamos el aviso de los estudiantes cada que terminaran una parte de la secuencia, en este caso, cuando terminaran la parte del inicio, desarrollo o cierre. El conductor de la secuencia cada tanto preguntaba si había dudas, algunos presentaban comodidad al resolver las actividades, otros preguntaron cosas esenciales acerca de lo que se pedía en la actividad.

De esa forma nos dimos cuenta que había que precisar las instrucciones y textos de apoyo que propusimos. La primera sección de inicio abordaba un tema general adentrando al estudiante a la problemática, y en el desarrollo ya implicaba realizar operaciones, tales como la regla de tres. Al llegar a la actividad de cierre fue donde se presentaron más problemas, ya que la redacción de las indicaciones no eran del todo claras, además que no precisamos los parámetros que queríamos que ellos escribieran, como: "pequeño", "grande", "muy pequeño" o "muy grande", y recibimos adjetivos comparativos de todo tipo. Esto no afectó en demasía los resultados, pero sin duda era algo que había que modificar. Al término de esa secuencia, se hizo una intervención para reportar comportamientos, dudas, expectativas, y platicar sobre el tema, ya que la mayoría parecía desconcertado al leer la última pregunta de dicha secuencia que era: “Cómo podríamos hacer una cantidad más pequeña si esta es infinitamente pequeña?" El término infinitamente pequeña había causado un tipo de desconcierto en los estudiantes y nadie parecía estar muy seguro de qué significado tenía eso.

Al culminar con la charla anterior, se entregaron las hojas de trabajo contestadas por medio de un correo electrónico y una vez entregadas, se repartieron las hojas de trabajo de la siguiente secuencia. La dinámica fue la misma, los estudiantes contestaron las hojas de trabajo de diferentes modos, unos en línea, otros la descargaron y la contestaron y otros mandaron fotos de sus procedimientos.

Al término del primer inciso de la actividad de inicio escribimos una breve explicación de cómo se demostraba que no existía un número más pequeño que todos en el campo de los números reales, pero causó mucha confusión ya que los estudiantes no sabian interpretar los símbolos matemáticos, por lo que hubo una intervención en ese momento, dicho momento que se aprovechó para institucionalizar a las magnitudes infinitamente pequeñas y sus propiedades que los definían como tal. Estas propiedades eran las que se simbolizaban con la letra $\delta$ y que cumplían con las siguientes características $\delta>0$ y $\delta^{2}=0$. Dicho esto, ellos pudieron continuar con las actividades.

Poco más tarde, nuevamente se encontraban con información que nosotros creímos sería pertinente para ellos a la hora de contestar, como lo siguiente: " $a<b \rightarrow a^{2}<b^{2} \leftrightarrow a>1$. Si $x<$ 
$\delta$, entonces $x^{2}<\delta^{2} \rightarrow 0<x^{2}<0$. Por lo que $x$ no existe". De tal manera que los estudiantes pudiesen concebir que no había un número más cercano al cero que un infinitesimal, pero causó una confusión importante en ellos, pues les era complicado entender que hablábamos de otro tipo de magnitud, y que estas magnitudes no se parecían a las magnitudes con las que habían tratado antes. Estas mismas complicaciones se notaron en las hojas de trabajo, ya que a los estudiantes les costaba concebir que la distancia entre un infinitesimal y otro, era una distancia infinitesimal, así como que podía haber más un infinitesimal en un mismo lugar de la recta en este caso.

Cuando empezaron la etapa del desarrollo de la segunda secuencia los estudiantes se enfrentaron a la adición de los infinitesimales, lo cual también fue complicado para ellos, debido a que las propiedades gráficas no habían quedado del todo claras, en el desarrollo cuando tuvieron que graficar las adiciones infinitesimales, tomaron al valor como mayor, pero lo situaron es una posición distinta, es decir, decían que $1+\delta>1+\delta^{2}$, lo cual no era verídico. Con ayuda de ese resultado, notamos que había que hacerse más énfasis en las propiedades gráficas de los infinitesimales. Acto seguido se presenta un texto de los que hemos estado hablando para ayudar al estudiante en su actividad posterior, en donde se indicaba la relevancia de la multiplicación de un número natural con un número infinitesimal, diciendo que este producto se convertía en un infinitesimal. Se presentaron prácticas matemáticas personales diferentes a las de referencia en lo que respecta a la propiedad distributiva a la hora de la multiplicación de naturales con infinitesimales, así como hubo complicación a la hora de graficar en la recta numérica dichos productos.

Por último, la actividad de cierre trataba de una figura geométrica, en este caso un cuadrado de lado $l$, el cual planteaba incrementos de medida precisa y un incremento de medida mínima $(\delta)$. Por motivos de familiarización, optamos por llamar $x$ al valor de $l$, y así pudiesen hacer sus representaciones algebraicas como acostumbraban. La actividad culminaba con lectura de uno de los textos de apoyo para institucionalizar a los incrementos mínimos como incrementos infinitesimales, y hacer una última actividad de esa secuencia, donde tenían que graficar geométricamente los incrementos de $2 \mathrm{~cm}$ y de $\delta \mathrm{cm}$. En este caso observamos algunas cosas, como que no hubo una aplicación de productos notables, sí hubo una aplicación de la propiedad $\delta^{2}=0$ en la representación algebraica, pero no una relación del símbolo $\delta$ con una variable para la modelación del incremento de lado de la figura, ni tampoco hubo incrementos gráficos mostrando los incrementos $\delta$ por la mayoría de los participantes. Terminó la sesión, se realizó una cita para el próximo día y mandaron sus hojas de trabajo. 


\section{Análisis}

Debido al tipo de trabajo que estamos desarrollando decidimos reportar solamente el análisis epistémico de la propuesta, por lo que a continuación presentaremos el contraste del análisis a priori y a posteriori de nuestra propuesta.

\subsection{A priori}

Los elementos del análisis a priori de la idoneidad epistémica de la propuesta son los siguientes:

- Se proponen situaciones variacionales y covariacionales para que el estudiante se problematice, además de que hacemos presente el significado polisémico de $\delta$ y los diferenciales.

- Se utilizan diferentes tipos de lenguaje en los problemas, así como conversiones entre estos lenguajes.

- Se presentan las definiciones, procedimientos y proposiciones fundamentales según el significado de referencia de las magnitudes infinitamente pequeñas y diferenciales.

- Se identifican y articulan los diversos significados promovidos por la secuencia.

\subsection{A posteriori}

A continuación se presentan algunos puntos de valoración del trabajo desarrollado por los estudiantes, incluyendo de ser necesario respuestas a las situaciones planteadas y las respectivas recomendaciones con respecto el análisis a posteriori de la idoneidad epistémica.

- Con base en las dudas y comentarios de los estudiantes en las diferentes discusiones surgidas durante las sesiones, pudimos notar que las situaciones problema planteadas resultaron satisfactorias ya que dichas dudas eran referentes a los diferentes contextos de los problemas y con la intención de resolverlos.

- Los lenguajes que intervienen en las secuencias varían entre el geométrico, algebraico, numérico y gráfico. Del mismo modo que emergen conversiones entre lenguajes.

- Durante la resolución de problemas en las secuencias escribimos estratégicamente breves notas informativas que ayudaban al estudiante con la construcción del significado pretendido. Las hojas de trabajo fueron el instrumento para observar el significado personal declarado de los estudiantes. Observamos el desarrollo de los diferentes 
procedimientos para la resolución de problemas, así como la aplicación de proposiciones y conceptos.

- La propuesta didáctica estaba diseñada para que el estudiante pudiera desarrollar sus sistemas de prácticas de tal manera que los conocimientos construidos en secuencias previas ayudaran a la resolución de secuencias siguientes. Con base en lo anterior, las diferentes interacciones y culminación de las secuencias de dicha propuesta, fueron elementos suficientes para afirmar que los significados pretendidos por la secuencia se alcanzaron.

En las Figuras 1 y 2 presentamos ejemplos de las diferentes prácticas de los estudiantes en sus hojas de trabajo. Decidimos mostrar solamente un breve fragmento de una misma actividad con la intención de mostrar únicamente las diferentes construcciones de significado personal declarado. En la Figura 3 presentamos un ejemplo las hojas de trabajo de un estudiante que resolvió los últimos problemas de nuestra propuesta utilizando el significado institucional pretendido construido como se esperaba.

Figura 1: Hoja de trabajo de la actividad de cierre de la secuencia didáctica 1 del estudiante 1

5. ¿Cómo es el peso de un camarón comparado con el peso de 5 tiburones? Muy pequeño.

6. ¿Cómo es el peso de un ratón comparado con el peso de una ballena azul? Muy pequeño.

7. ¿Cómo es el peso de un alga comparado con el peso del Tule? Muy pequeño.

8. ¿Cómo es el peso de una hormiga comparado con el peso de 10 elefantes? Muy pequeño.

9. ¿Cómo es el peso de un microbio comparado con el peso de 20 ballenas azules? Insignificante.

10. ¿Cómo es el peso de una partícula subatómica comparado con el peso de Júpiter? Absurdamente pequeño. 
Figura 2: Hoja de trabajo de la actividad de cierre de la secuencia didáctica 1 del estudiante 2

5. ¿Cómo es el peso de un camarón comparado con el peso de 5 tiburones? Es una gran diferencia por que el camarón el algo muy pequeño y los 5 tiburones son muy grandes.

6. ¿Cómo es el peso de un ratón comparado con el peso de una ballena azul? Es una diferencia gigante, ella ballena es muv grande y el ratón muv pequeño.

7. ¿Cómo es el peso de un alga comparado con el peso del Tule? Es una diferencia bastante grande.

8. ¿Cómo es el peso de una hormiga comparado con el peso de 10 elefantes? Es una diferencia gigante, la hormiga es demasiado pequeña a comparación.

9. ¿Cómo es el peso de un microbio comparado con el peso de 20 ballenas azules? Es una diferencia exageradamente grande, lo pequeño de uno con lo gigante del otro.

10. ¿Cómo es el peso de una partícula subatómica comparado con el peso de Júpiter? Es una diferencia prácticamente infinita por que los tamaños ni se pueden comparar.

Fuente: Félix (2020).

Figura 3: Hoja de trabajo de la actividad de desarrollo de la secuencia didáctica 6 de estudiante 3

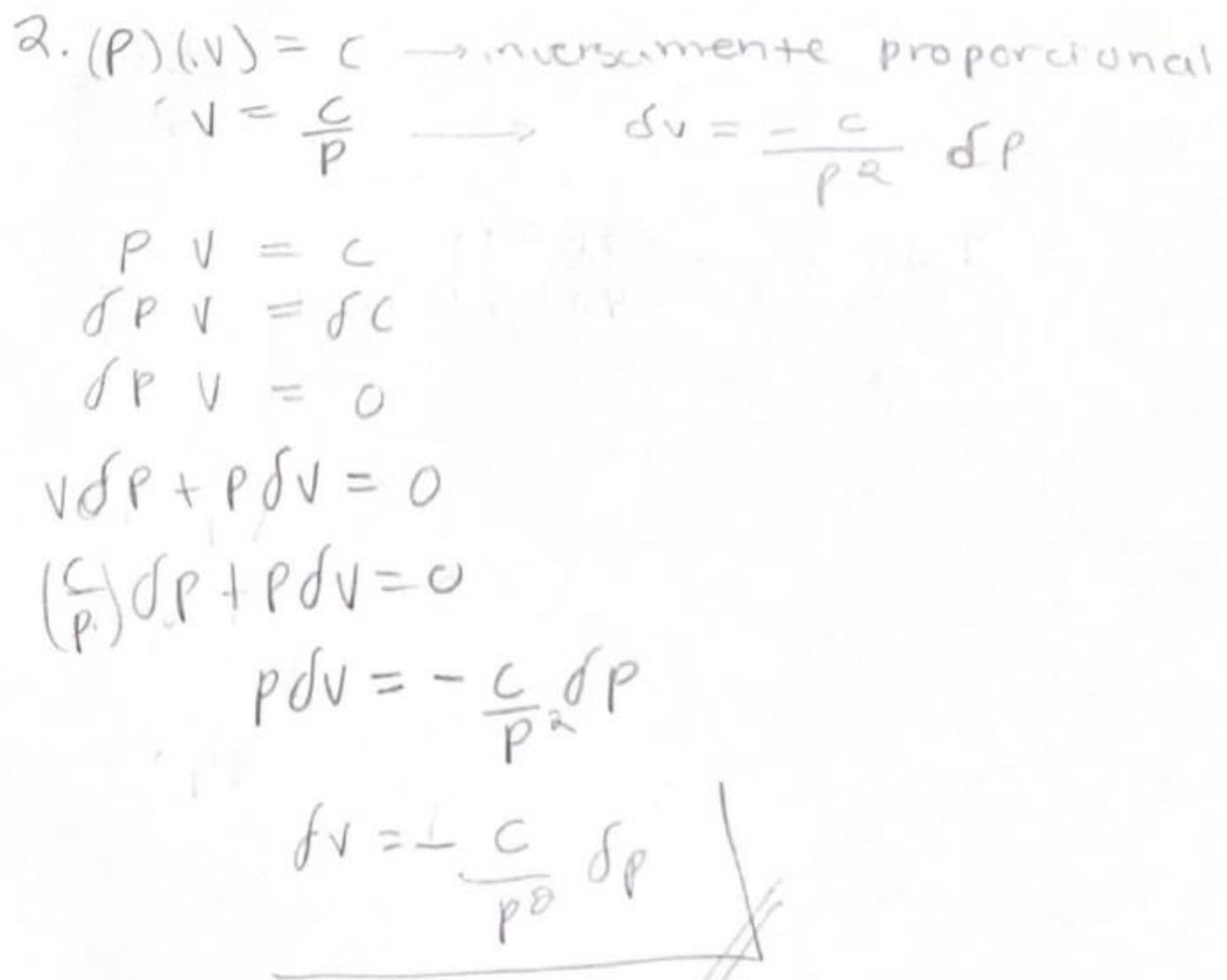

Fuente: Félix (2020). 


\section{Conclusiones}

Con base en lo descrito y en las experiencias de la implementación, se pudo notar que los conceptos de muy grande, grande, pequeño y muy pequeño son complicados de caracterizar de debido a la relatividad de las cantidades, con base en las comparaciones que planteamos en cada situación problema. Del mismo modo, se presentaron dificultades con los términos como infinito, infinitamente pequeño, y la acción de operar con magnitudes de dimensión inasignable.

A pesar de las circunstancias presentadas, los estudiantes tuvieron un desempeño suficientemente satisfactorio, por lo que hubo condiciones adecuadas para la observación de resultados; las dudas surgidas, la diversidad de respuestas y las concepciones de estos fueron piezas claves para realizar un análisis descriptivo de nuestra propuesta. Si bien el concepto límite conlleva diversas dificultades, la propuesta diferente que nosotros desarrollamos con base en las magnitudes infinitamente pequeñas no está exenta de dificultades.

Dado que el análisis a priori que realizamos se corresponde con el significado institucional pretendido, pues la base para realizarlo son las respuestas que nosotros dimos a las situaciones problemas planteadas, las respuestas de los estudiantes que constituyen la base para el análisis a posteriori se corresponden con sus significados personales. La parte que mostramos en este artículo nos permite concluir entonces que los significados personales construidos por los estudiantes se corresponden con los significados institucionales pretendidos, lo cual en general es verdadero, pero también es necesario señalar que existen aspectos de la secuencia en los que esta situación es ligeramente distinta y la contrastación entre los significados personales construidos (análisis a posteriori) y los significados institucionales pretendidos (análisis a priori) nos proporcionó elementos para hacer modificaciones al diseño de nuestra secuencia didáctica, lo cual es necesario reportar con mayor detenimiento en otra oportunidad.

\section{Referencias}

ARTIGUÉ, M.; DOUADY, R.; MORENO, L.; GÓMEZ, P. La enseñanza de los principios del cálculo: problemas epistemológicos, cognitivos y didácticos. Ingeniería didáctica en educación matemática.1995.

BARRIOSETA, L. Derivadas de la vida cotidiana. Tesis de maestría sin publicar, Universidad de La Rioja. 2014.

BELL, J. A primer of infinitesimal analysis. Cambridge University Press. 1998.

CORNU, B. Apprentissage de la notion de limite: modèles spontanés et modèles propes. Proceedings PME-V, Grenoble, France, Vol. I, 322-326.1981. 
DANHKE, G. Investigación y comunicación. En C. Fernández-Collado y G. L. Danhke (Eds.). La comunicación humana: Ciencia social. México: McGraw-Hill. 1989, p. 385-454.

FÉLIX, G. Cantidades infinitamente pequeñas y razón instantánea de cambio. Tesis de maestría sin publicar, Universidad de Sonora. 2020.

GODINO, J. D. Indicadores de la idoneidad didáctica de procesos de enseñanza y aprendizaje de las matemáticas. Cuadernos de Investigación y Formación en Educación Matemática, 111132, 2013.

GODINO, Juan. Hacia una teoría de la instrucción matemática significativa. 2002. Documento de trabajo del curso de doctorado "Teoría de la educación Matemática". Universidad de Granada.

GODINO, J. D. et al. Enfoque ontosemiótico de los conocimientos y competencias del profesor de matemáticas. Bolema, 31 (57), 90-113. 2017.

GODINO, J. D. Un enfoque ontológico y semiótico de la cognición matemática. Recherches en Didactiques des Mathematiques, 22 (2/3), 237-284. 2002.

GODINO, J. D. et al. Una perspectiva ontosemiótica de los problemas y métodos de investigación en educación matemática. Revemop, v. 3, p. e202107, 21 jun. 2021. DOI: https://doi.org/10.33532/revemop.e202107

GODINO, J.; BATANERO, C. y FONT V. Un enfoque ontosemiótico del conocimiento y la instrucción matemática. 2009. Recuperado de: https://www.ugr.es/ igodino/funcionessemioticas/sintesis_eos_10marzo08.pdf.

GONZÁLEZ, L.; RADILLO, M. Una propuesta para la enseñanza del concepto de derivada de una función, mediante actividades de visualización. ALME, 27, 925-932. 2014.

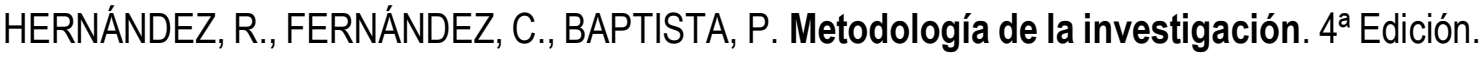
Editorial McGrawHill. Ciudad de México. 2006.

SCHIVO, M.; SGRECCIA, N.; CALIGARIS, M. Derivada y aplicaciones: la tecnología en el aula. ALME, 27, 2075-2083. 2014.

VERÓN, M. A.; GIACOMONE, B. Análisis de los significados del concepto de diferencial desde una perspectiva ontosemiótica. Revemop, v. 3, p. e202109, 22 jun. 2021. DOI: https://doi.org/10.33532/revemop.e202109 